\title{
Electron-phonon vertex in the two-dimensional one-band Hubbard model
}

\author{
Z. B. Huang, W. Hanke, and E. Arrigoni \\ Institut für Theoretische Physik, Universität Würzburg, am Hubland, 97074 Würzburg, Germany \\ D. J. Scalapino \\ Department of Physics, University of California, Santa Barbara, California 93106-9530 USA
}

(Dated: October 16, 2018)

\begin{abstract}
Using quantum Monte Carlo techniques, we study the effects of electronic correlations on the effective electron-phonon (el-ph) coupling in a two-dimensional one-band Hubbard model. We consider a momentum-independent bare ionic el-ph coupling. In the weak- and intermediate-correlation regimes, we find that the on-site Coulomb interaction $U$ acts to effectively suppress the ionic elph coupling at all electron- and phonon- momenta. In this regime, our numerical simulations are in good agreement with the results of perturbation theory to order $U^{2}$. However, entering the strong-correlation regime, we find that the forward scattering process stops decreasing and begins to substantially increase as a function of $U$, leading to an effective el-ph coupling which is peaked in the forward direction. Whereas at weak and intermediate Coulomb interactions, screening is the dominant correlation effect suppressing the el-ph coupling, at larger $U$ values irreducible vertex corrections become more important and give rise to this increase. These vertex corrections depend crucially on the renormalized electronic structure of the strongly correlated system.
\end{abstract}

The role of the el-ph interaction in the physics of the high $T_{c}$ cuprate superconductors remains unclear. On the one hand, the linear $T$ dependence of the resistivity up to high temperatures and the small value of the isotope coefficient of the optimally doped materials suggest that the el-ph interaction plays a secondary role $\stackrel{1}{\underline{1}}$. The fact that the undoped cuprates are Mott antiferromagnetic insulators supports the notion that strong Coulomb interactions are dominant and that the essential physics is contained in the Hubbard and t-J models ${ }^{2}$. On the other hand, however, a variety of experiments also display pronounced phonon and electron-lattice effects in these materials: superconductivity-induced phonon renormalization $^{3}$, large isotope coefficients away from optimal doping ${ }^{4}$, tunneling phonon structures $\underline{\underline{5}}$, etc., give evidence of significant el-ph coupling. Recently, photoemission data indicated a sudden change in the electron dispersion near a characteristic energy scale ${ }^{6}$, which is possibly caused by coupling of electronic quasiparticles to phonon modes.

To elucidate the effects of strong electronic correlations on the el-ph interaction, several authors have calculated the el-ph vertex function in the one- and three-band Hubbard models based on $(1 / N)$ expansion within slave$\operatorname{boson}^{7.8}$ and $\mathrm{X}$ operator ${ }^{\underline{9}}$ formalisms. One finding ${ }^{9}$ is that for the ionic, i.e. onsite, el-ph coupling in the underdoped regime, the backward scattering with large phonon momentum transfer is suppressed much more than the forward scattering with small phonon momentum transfer. Based on this finding, it was argued that a forward peaking of the renormalized el-ph vertex could account for the absence of phonon features in the transport data. In addition, an el-ph interaction which is peaked at small phonon momentum transfer contributes to an attractive interaction in the $d_{x^{2}-y^{2}}$-pairing channe ${ }^{9,10}$. One should notice that these previous calculations were limited by the approximate nature of $1 / N$ and slave-boson treatments, moreover, they were carried out for $U \rightarrow \infty$.
Here, we would like to use a more accurate numerical method to gain further insight into the way in which strong electron correlations dress the el-ph coupling for a range of values of $U$ from weak to strong correlation. This analysis is important since it turns out that the $U$ dependence of the el-ph coupling shows a dramatic change as a function of $U$ (see below). Specifically, we apply the determinantal Monte Carlo 11 algorithm to find the single-particle response to external phonon fields in the one-band Hubbard model. In particular, we will calculate an effective el-ph coupling ${ }^{12} g(p, q)$ (effective el-ph vertex) for scattering quasiparticles near the Fermi surface (which includes screening, vertex corrections, and the quasiparticle renormalization) produced by the Hubbard $U$.

Our principle findings are: (1) Initially (up to the value of $U \approx 6 t$ ), as the Hubbard-Coulomb interaction $U$ increases, the el-ph coupling is suppressed by electronic correlations for all phonon and electron momenta, and in particular for the backward scattering around $\mathbf{q}=(\pi, \pi)$. The suppression is due to the conventional screening term. (2) The behavior changes even qualitatively in the strong-correlation regime $(U \geq 6 t)$. Here, the effective el-ph coupling at small phonon momentum transfer increases with increasing $U$, while the one at large phonon momentum transfer appears to saturate (see Fig. 3). The increase of the el-ph coupling in the forward direction is due to irreducible vertex corrections, which become the dominant correlation effects at strong Coulomb interactions. These vertex corrections are intimately connected with the renormalized electronic structure. It is argued that the picture of a "spin-bag" quasiparticle can explain the qualitatively different el-ph couplings for small and large phonon momenta. Furthermore, we would like to stress that our numerical results for the charge compressibility, which decreases monotonically with increasing $U$, rule out the explanation of the increase of the el-ph ver- 
tex at small $\mathbf{q}$ as a function of $U$ in terms of a close-by phase separation or charge instability 13 .

Our starting point is the one-band Hubbard model,

$$
H=-t \sum_{\langle i j\rangle, \sigma}\left(c_{i \sigma}^{\dagger} c_{j \sigma}+c_{j \sigma}^{\dagger} c_{i \sigma}\right)+U \sum_{i} n_{i \uparrow} n_{i \downarrow},
$$

The operators $c_{i \sigma}^{\dagger}$ and $c_{i \sigma}$ as usual create and destroy an electron with spin $\sigma$ at site $i$, respectively and the sum $\langle i j\rangle$ is over nearest-neighbor lattice sites. Here, $U$ is the onsite Coulomb interaction and we will choose the nearest-neighbor hopping $t$ as the unit of energy.

In our simulations, we have used the linear-response technique in order to extract the el-ph vertex function. In this method, one formally adds to Eq. (1) the interaction with a momentum- and (imaginary) time-dependent lattice distortion (phonon) field $u_{\mathbf{q}} e^{-i q_{0} \tau}$ in the form ${ }^{12.14}$

$$
H_{e l-p h}=\sum_{\mathbf{k q} \sigma} g_{\mathbf{k q}}^{0} c_{\mathbf{k}+\mathbf{q} \sigma}^{\dagger} c_{\mathbf{k} \sigma} u_{\mathbf{q}} e^{-i q_{0} \tau},
$$

where $g_{k q}^{0}$ is the bare el-ph coupling. One then considers the "anomalous" single-particle propagator in the presence of this perturbation defined as ${ }^{12}$

$G_{A}(p, q) \equiv-\int_{0}^{\beta} d \tau e^{i\left(p_{0}+q_{0}\right) \tau}\left\langle T_{\tau} c_{\mathbf{p}+\mathbf{q} \sigma}(\tau) c_{\mathbf{p} \sigma}^{\dagger}(0)\right\rangle_{H+H_{e l-p h}}$,

where \langle\rangle$_{H+H_{e l-p h}}$ is Green's function evaluated with the Hamiltonian $H+H_{e l-p h}$. Diagrammatically $G_{A}(p, q)$ has the structure shown in Fig. I so that the el-ph vertex function $\Gamma(p, q)$ can be expressed quite generally in terms of $G_{A}$ and of the single-particle Green's function $G(p)$ in the form

$$
\Gamma(p, q)=\lim _{u_{\mathbf{q}} \rightarrow 0} \frac{1}{u_{\mathbf{q}}} \frac{G_{A}(p, q)}{G(p+q) G(p)},
$$

It is, thus, sufficient to calculate the leading linear response of $G_{A}$ to $H_{\mathrm{el}-\mathrm{ph}}$, which is given by

$$
\begin{aligned}
G_{A}(p, q)= & u_{\mathbf{q}} \int_{0}^{\beta} d \tau e^{i\left(p_{0}+q_{0}\right) \tau} \int_{0}^{\beta} d \tau^{\prime} e^{-i q_{0} \tau^{\prime}} \sum_{\mathbf{k q} \sigma^{\prime}} g_{\mathbf{k q}}^{0} \times \\
& \left\langle T_{\tau} c_{\mathbf{k}+\mathbf{q} \sigma^{\prime}}^{\dagger}\left(\tau^{\prime}+0^{+}\right) c_{\mathbf{k} \sigma^{\prime}}\left(\tau^{\prime}\right) c_{\mathbf{p}+\mathbf{q} \sigma}(\tau) c_{\mathbf{p} \sigma}^{\dagger}(0)\right\rangle_{H},(5)
\end{aligned}
$$

where $0^{+}$is a positive infinitesimal. The two-particle Green's function in Eq. (5) is evaluated with respect to the pure Hubbard Hamiltonian (Eq. (1)). Since we have only considered the linear-response contribution from the phonon field, the el-ph vertex $\Gamma$ contains full contributions from Coulomb interactions only 15 ,

Close to the Fermi energy, the single-particle Green's function can be written as

$$
G(p)=\frac{1}{Z(p)\left(i p_{0}-E_{\mathbf{p}}\right)},
$$

where $Z(p)$ is the wave-function renormalization and $E_{\mathbf{p}}$ the quasiparticle excitation energy $\stackrel{16}{\underline{16}}$. Then for electron

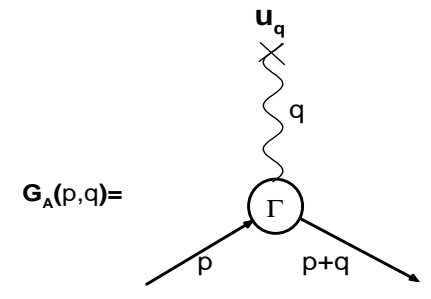

FIG. 1: Diagrammatic representation of $G_{A}(p, q)$ within linear response to $u_{\mathbf{q}}$. The thick solid lines represent dressed single-particle Green's functions of the Hubbard model. The wavy line denotes the external perturbation in Eq. (2).

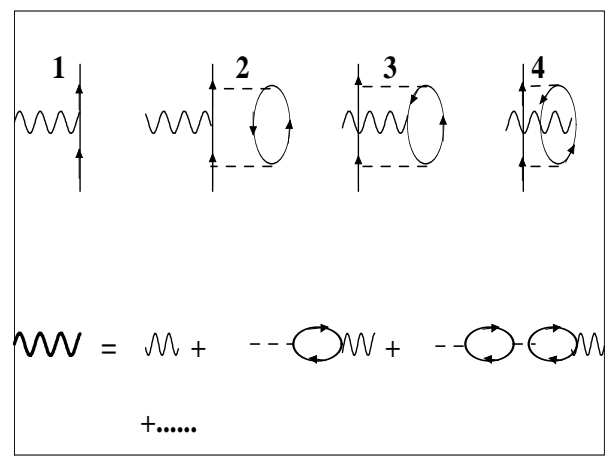

FIG. 2: Low-order Feynman diagrams for the irreducible el-ph vertex $\Lambda(p, q)$ (top) and low-order polarization graphs (lower) that enter the full vertex $\Gamma$. The thin solid lines are the noninteracting Green's functions and the dashed lines represent the Hubbard interaction $U$. The thin (thick) wavy line stand for the bare (screened phonon) fields.

scattering processes which involve states near the Fermi surface, the effective el-ph coupling reads

$$
g(p, q)=\frac{\Gamma(p, q)}{\sqrt{Z(p) Z(p+q)}}
$$

In the following, we will focus on the case of an ionic el-ph coupling, in which the bare coupling $g_{\mathbf{p q}}^{0}$ is a constant $g^{0}$. Since we are considering linear terms in $g^{0}$ only, we can set $g^{0}$ equal to 1 . This corresponds to the simple Holstein form of the el-ph interaction, which is an important limiting case. Moreover, having the bare interaction independent of $\mathbf{p}$ and $\mathbf{q}$ makes it easier to see modifications, which arise from the strong $U$ correlation effects.

The low order $U$ and $U^{2}$ vertex contributions to $\Gamma$ are displayed in Fig. 2] The diagrams shown at the bottom of Fig. 2 are the leading terms of the RPA approximation $\left(1-\frac{1}{2} U \Pi_{0}(q)\right)^{-1}$ to the polarization correction, with $\Pi_{0}(q)$ the contribution from the single bubble. The exact Monte Carlo result for the polarization correction is 
$1+\frac{1}{2} U \Pi(q)$ with

$$
\Pi(q)=-\int_{0}^{\beta} d \tau e^{-i q_{0} \tau}\left\langle T_{\tau} \rho_{\mathbf{q}}(\tau) \rho_{\mathbf{q}}^{\dagger}(0)\right\rangle,
$$

and

$$
\rho_{\mathbf{q}}^{\dagger}=\frac{1}{\sqrt{N}} \sum_{\mathbf{k} \sigma} c_{\mathbf{k}+\mathbf{q} \sigma}^{\dagger} c_{\mathbf{k} \sigma}
$$

With this in mind, $\Gamma$ can be written in terms of the screening factor and an irreducible vertex $\Lambda(p, q)$, which is the sum of graphs that can not be separated into two pieces by cutting a single dashed Coulomb interaction line $U$ (see Fig. 2), i.e.

$$
\Gamma(p, q)=\left(1+\frac{1}{2} U \Pi(q)\right) \Lambda(p, q),
$$

Thus, the strong-correlation effects associated with the Hubbard-Coulomb interaction $U$ lead to an effective elph coupling which can be expressed in the canonical form

$$
g(p, q)=\frac{\left(1+\frac{1}{2} U \Pi(q)\right) \Lambda(p, q)}{(Z(p) Z(p+q))^{\frac{1}{2}}} .
$$

and one sees that it consists of a product of an irreducible vertex $\Lambda(p, q)$, a screening factor $\left(1+\frac{1}{2} U \Pi(q)\right)$, and a quasiparticle renormalization $(Z(p) Z(p+q))^{-\frac{1}{2}}$ factor.

Our numerical Monte Carlo simulations were performed on an $8 \times 8$ lattice at an inverse temperature $\beta=2$ and a filling $\langle n\rangle=0.88$. We have set the frequencies to their minimum values, i.e., $p_{0}=\pi T$ for fermions and $q_{0}=0$ for bosons. We have checked some special cases for which one can reach lower temperatures, namely a $2 \mathrm{D}$ system at weak correlation and/or with large doping $(<n\rangle=0.65)$. For these systems, we have found that the real part of the vertex function $\Gamma((\pi T, \mathbf{p}),(0, \mathbf{q}))$ depends only weakly on temperature, and the imaginary part always vanishes as $T \rightarrow 0$. In the following we will, therefore, focus on the real part of the vertex function at $p_{0}=\pi T$. Comparison with exact diagonalization on a 4-site ring demonstrates that the difference in $\Gamma(p, q)$ between the two results is less than two percent up to $U=8$.

We are interested in el-ph scattering processes in which the incoming and the outgoing electron momenta $\mathbf{p}$ and $\mathbf{p}+\mathbf{q}$ are close to the Fermi surface. For an $8 \times 8$ lattice doped near half-filling, the $q$ and $U$ dependence of $g(p, q)$ for the scattering processes on the half-filled diamond Fermi surface is studied. In particular, we will examine initial states corresponding to $\mathbf{p}=(-\pi, 0)$ and $\mathbf{p}=(-\pi / 2, \pi / 2)$. Other choices of $\mathbf{p}$ and $\mathbf{p}+\mathbf{q}$ close to the half-filled diamond Fermi surface give qualitatively similar results to those reported here.

Monte Carlo results for $g(p, q)$ and for the polarization factor $\left(1+\frac{1}{2} U \Pi\right)$ are shown in Fig. 3 The left (a)

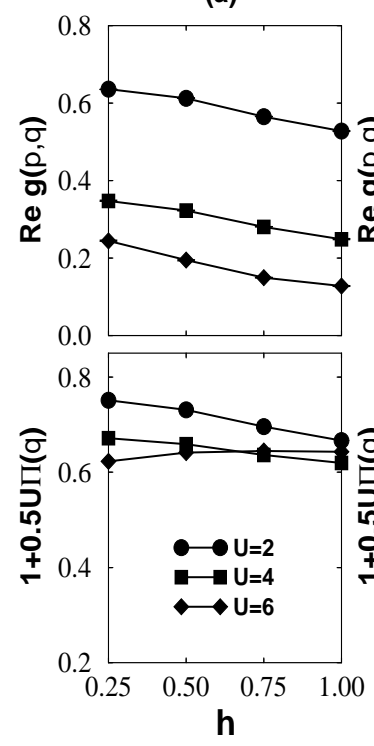

(b)
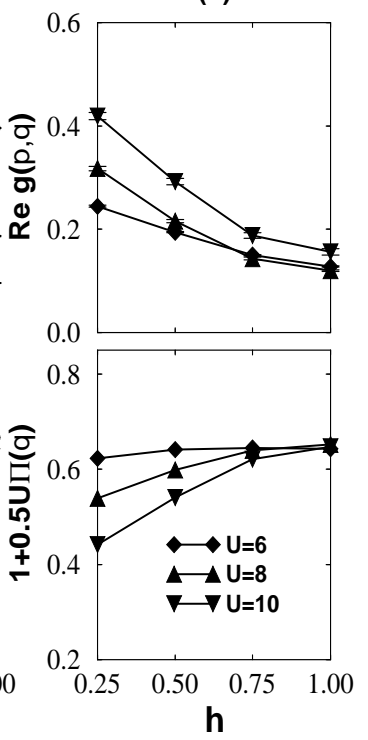

FIG. 3: Real part of the effective el-ph coupling $g(p, q)$ and the polarization factor $1+\frac{1}{2} U \Pi$ versus $\mathbf{q}$ for (a) $U \leq 6$ and (b) $U \geq 6$. Here $\mathbf{q}=\pi(h, h)$ with $h$ the tick label of the $x$ axis. The incoming electron carries momentum $\mathbf{p}=(-\pi, 0)$ and the value of $U$ is indicated by the shape of the symbol.

hand side, Fig. 3a, shows the behavior in the weak- and intermediate-correlation regimes. The right hand side of the figure, Fig. 30, shows similar results when the system enters the strong-correlation regime. One can clearly see that when the Hubbard $U$ is smaller than $U \approx 6, g(p, q)$ decreases as a function of $U$ from its bare value $g^{0}=1$, for all momentum transfers. Then, as the interaction $U$ increases to the strong-correlation case $(U \sim W=8 t)$, the effective el-ph coupling begins to increase. This behavior is particularly evident at smaller values of momentum transfer. Our finding at large phonon momentum is similar to that of Deppeler et al.'s work ${ }^{17}$, which shows that the local el-ph interaction is suppressed by both electronic correlations and dynamic phonon vertex corrections. In the strong-correlation regime, the overall $\mathbf{q}$ dependence of the el-ph coupling agrees reasonably well with the results of the $1 / N$ expansion ${ }^{9}$ which are obtained for the $U \rightarrow \infty$ limit. However, in our case the interesting behavior is that the effective el-ph coupling as a function of $U$ is nonmonotonic, first decreasing and then, at physically interesting values of $U$, increasing. This finding deviates from the prediction of a Fermi-liquid analysis ${ }^{8}$. According to this analysis, $\lim _{\mathbf{q} \rightarrow 0, q_{0}=0} g(p, q) \propto \frac{1}{1+F_{0}^{s}}$ with $F_{0}^{s}$ the zero-harmonic symmetric Landau amplitude so that the effective el-ph coupling decreases monotonically with increasing $U$ since $F_{0}^{s}$ becomes larger with $U$ (except when approaching a charge instability).

From Fig. 3 one can see that the polarization factor acts quite generally to suppress the el-ph coupling. At large momentum transfer, this quantity saturates as $U$ 


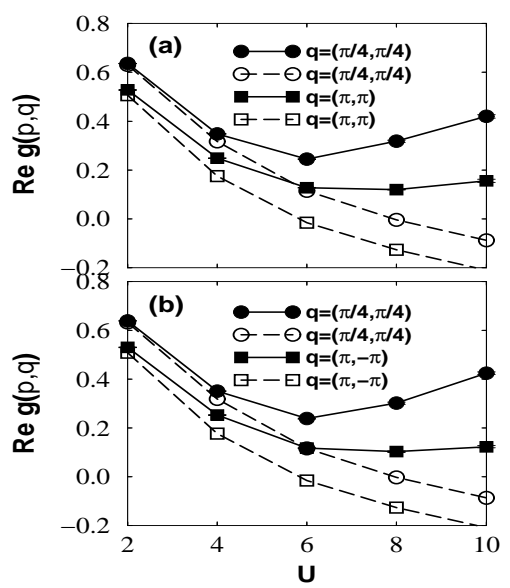

FIG. 4: Real part of $g(p, q)$ as a function of $U$ for (a) $\mathbf{p}=$ $(-\pi, 0)$ and (b) $\mathbf{p}=(-\pi / 2, \pi / 2)$. The value of $\mathbf{q}$ is indicated by the shape of the symbol. The solid circles are Monte Carlo results and the open symbols show the perturbation theory contributions shown in Fig. 2

increases, while at small momentum transfer it continues to decrease. Comparison between $g(p, q)$ and the polarization factor indicates that at weak correlation screening is the dominant correlation effect suppressing the el-ph coupling. On the other hand, with an increasing Hubbard $U$, the vertex corrections $\Lambda$ become more important, making the effective el-ph coupling peaked in the forward scattering direction.

In order to see the $U$ dependence more clearly, in Fig. 4 Quantum Monte Carlo calculations are compared with perturbation theory for different values of $U$. Here, the solid symbols are Monte Carlo results and the open symbols show the results obtained by evaluating $\Gamma(p, q)$ perturbatively with the diagrams of Fig. 2. In the perturbative calculations, $g(p, q)$ is calculated by using wavefunction renormalizations $Z(p)$ and $Z(p+q)$ extracted from Monte Carlo data. As one can see, in the weakcorrelation regime, the perturbative calculations are in good agreement with Monte Carlo simulations. However, when the Hubbard $U$ exceeds $U \approx 4\left(\sim \frac{W}{2}\right)$, perturbation theory appears to break down.

QMC calculations of the doping, temperature and $U$ dependence of the vertex enhancement and its $\mathbf{q}$ dependence, which will be presented in more detail in a longer paper, give a physical picture for both the unexpected increase of the vertex as a function of larger $U$-values for small phonon momenta and also the suppression of the vertex for large phonon momenta: It is well estab- lished, in particular in terms of QMC work on the singleparticle spectral function of the Hubbard model18, that the single-particle excitations as a function of increasing $U$-values undergo (at lower enough temperatures, around $\beta=2-3$ ) a crucial physical transition into a strong-correlation regime around $U \approx 6 t$ : the electronically filled valence band of width $W$, which is essentially given by the bare band width $W=8 t$, splits into two "bands". The physical picture behind this splitting is the formation of a "spin-bag" quasiparticle, i.e. of the bare particle (hole) dressed with a spatially (typically a few lattice constants) extended spin cloud, which is due to the frustration of the local antiferromagnetic order. The spin bag moves coherently and "slowly" with an energy scale $J=\frac{4 t^{2}}{U}$ within the new, strongly renormalized quasiparticle band of width $\sim J$. This coherent motion couples effectively (with small energy denominators) to longer wavelength lattice displacements, whose wavelength is typically longer than the spin-bag "diameter". On the other hand, there is at larger $U$-values also an incoherent lower Hubbard band whose higher energy scale corresponds to the "rattling around" of the bare particle within the spin bag ${ }^{18}$. Only large momenta phonons, i.e. with wavelength smaller than the "extension" of the spin bag, can couple to these incoherent electronic degrees of freedom. Their coupling is weak because of the combined effects of the incoherent motion and the large $(\sim$ scale $W)$ energy denominators.

In summary, based on QMC simulations, we have studied the el-ph vertex function in the two-dimensional Hubbard model. We find that in the weak-correlation regime, the effects of the Hubbard interaction $U$ are to suppress the ionic el-ph coupling at all phonon momenta, with backward scattering processes being more strongly suppressed than forward ones. On the other hand, in the strong-correlation regime, the vertex at smaller phonon momentum transfer anomalously increases as a function of $U$. We also find that screening is the dominant contribution to the vertex corrections at weak correlation, while at strong correlation the irreducible vertex corrections are crucial.

We would like to acknowledge useful discussions with Dr. R. Zeyher and C. Castellani. The Würzburg group would like to acknowledge support by the DFG under Grant No. Ha 1537/20-1 and by a Heisenberg Grant (AR 324/3-1), and by the Bavaria California Technology Center $(\mathrm{BaCaTeC})$, the KONWHIR projects OOPCV and CUHE. DJS acknowledges support from the US Department of Energy under Grant \#DOE85-45197. The calculations were carried out at the high-performance computing centers HLRS (Stuttgart) and LRZ (München).

\footnotetext{
1 M. Imada, A. Fujimori, and Y. Tokura, Rev. Mod. Phys. 70, 1039 (1998).
}

2 P.W. Anderson, cond-mat/0201429

${ }^{3}$ V.G. Hadjiev, X.J. Zhou, T. Strohm, M. Cardona, 
Q.M. Lin, and C.W. Chu, Phys. Rev. B 58, 1043 (1998); for a review, see also M.L. Kulic, Physics Reports 338, 1-264 (2000).

4 J.P. Franck, S. Harker, and J.H. Brewer, Phys. Rev. Lett. 71, 283 (1993).

5 D. Shimada, Y. Shiina, A. Mottate, Y. Ohyagi, and N. Tsuda, Phys. Rev. B 51, R16495 (1995).

6 A. Lanzara, P.V. Bogdanov, X.J. Zhou, S.A. Keller, D.L. Feng, E.D. Lu, T. Yoshida, H. Eisaki, A. Fujimori, K. Kishio, J.-I. Shimoyama, T. Noda, S. Uchida, Z. Hussain, and Z.-X. Shen, Nature 412, 510 (2001).

7 J.H. Kim, and Z. Tesanovic, Phys. Rev. Lett. 71, 4218 (1993).

8 M. Grilli and C. Castellani, Phys. Rev. B 50, 16880 (1994).

${ }^{9}$ R. Zeyher and M.L. Kulic, Phys. Rev. B 53, 2850 (1996).

10 N. Bulut and D.J. Scalapino, Phys. Rev. B 54, 14971 (1996).

11 R. Blankenbecler, D.J. Scalapino, and R.L. Sugar, Phys. Rev. D 24, 2278 (1981).

12 In our convention, unbolded variables denote both Matsubara frequency and momentum, i.e., $p=\left(p_{0}, \mathbf{p}\right)$ and $q=\left(q_{0}, \mathbf{q}\right)$. For a static phonon, $q_{0}$ is set to be zero.
13 B. Cerruti, E. Cappelluti, and L. Pietronero, cond-mat/0307190

14 The imaginary-time-dependent perturbation (2) has a well-defined meaning within a functional-integral formulation, where it leads to the linear-response result (5). Details will be given elsewhere.

15 This method could, of course, be extended to take into account phonon vertex corrections, but here we are interested in understanding the effects of the Hubbard interaction $U$ on the vertex.

16 Note that $E_{\mathbf{p}}$ is a complex variable due to lifetime effects. For simplicity, we have assumed $\operatorname{Im} E_{\mathbf{p}}<<p_{0}$ and extracted the wave-function renormalization $Z(p)$ from the equation $\operatorname{Im} \frac{1}{G(p)}=Z(p) p_{0}$.

17 A. Deppeler and A.J. Millis, Phys. Rev. B 65, 100301 (2002); ibid. 65, 224301 (2002); S. Blawid, A. Deppeler, and A.J. Millis, ibid. 67, 165105 (2003); J.K. Freericks, ibid. 50, 403 (1994); J.K. Freericks and M. Jarrell, ibid. 50, 6939 (1994).

18 C. Gröber, R. Eder, and W. Hanke, Phys. Rev. B 62, 4336 (2000), and references therein. 